\title{
Valoración de externalidades territoriales en denominaciones de origen de aceite de oliva mediante técnicas de Proceso Analítico de Red
}

\author{
L. Pérez y Pérez*,1, P. Egea** y J. Sanz-Cañada*** \\ * Centro de Investigación y Tecnología de Aragón. Avda. Montañana, 930. 50059 Zaragoza \\ ** Universidad de Zaragoza. Gran Vía, 2. 50005 Zaragoza \\ *** Centro de Ciencias Humanas y Sociales - C.S.I.C. Albasanz, 26-28 - 28037 Madrid
}

\section{Resumen}

Las producciones agroalimentarias locales llevan a cabo, además de su función comercial, otras funciones, cada vez más importantes para la sociedad, que se refieren a la generación de bienes públicos. Este trabajo valora las externalidades territoriales producidas por los Sistemas Agroalimentarios Locales (SIAL) de alimentos típicos, como son los vinculados a las denominaciones de origen protegidas (DOP). Se ha desarrollado una metodología de valoración de externalidades territoriales, destinada a su uso en el diseño de políticas públicas. Nuestro objeto de estudio es el aceite de oliva y las zonas para el análisis empírico son las DOP de Estepa (Sevilla y Córdoba) y Sierra de Segura (Jaén). Desde el punto de vista de la demanda de externalidades territoriales, el artículo aporta una metodología de valoración de externalidades en las DOP mediante técnicas de decisión multicriterio -Proceso Analítico de Red (ANP)- basadas en las opiniones reveladas por expertos y la subsiguiente obtención de consensos. Como avance metodológico, se incorporan a los análisis de demanda de externalidades agroambientales la demanda de otros bienes públicos, como los vinculados al origen, al capital social y al patrimonio cultural, con un enfoque transversal de análisis territorial. Los resultados indican que los criterios que más influyen en los esquemas de preferencias locales son la acción colectiva en materia de comercialización, la calidad diferencial y la creación de un entorno innovador.

Palabras clave: Sistemas agroalimentarios locales (SIAL), multifuncionalidad agraria, comercialización colectiva, calidad diferencial, entorno innovador, Proceso Analítico de Red (ANP).

\section{Abstract \\ Territorial externalities valuation in olive oil protected designations of origin using the Analytical Notwork Process}

Local agro-food production does not simply produce commercial goods, but it contributes increasingly to the supply of public goods. The aim of this work is to value the territorial externalities produced by the economic-institutional activities of Local Agro-Food Systems (LAFS) with respect to typical food products, such as those linked to protected designations of origin (PDOs). The purpose of the paper is to develop a methodology for the analysis and the valuation of non-market goods, in order to implement public policies. The project focuses on the olive oil sector. The field areas chosen are the PDOs of Estepa (Sevilla and Cordoba) and Sierra de Segura (Jaén). From the point of view of the demand for territorial externalities, the paper seeks to develop a methodology focusing on the valuation of non-market goods in PDOs, using multi-criteria decision techniques -Analytic Network Process (ANP)- based on the

1. Autor para correspondencia: Iperez@aragon.es http://dx.doi.org/10.12706/itea.2013.015 
opinions of experts and the subsequent methods for obtaining consensus. As a methodological innovation, the demand for other public goods has been incorporated to the demand for agri-environmental externalities, for example those related to the origin, to the social capital or to the cultural heritage, using a cross-section approach inherent to the territorial analysis. Results show that the most influencing criteria on the expert preferences schemes are the collective action in marketing, the differential quality, and the creation of an innovative environment.

Key words: Local Agro-Food Systems; agricultural multifunctionality; collective action in marketing, differentiation-related quality, innovative environment; Analytic Network Process (ANP).

\section{Introducción}

Las actividades agroalimentarias suministran, además de materias primas y alimentos, otros bienes y servicios valorados por la sociedad: desempeñan funciones ambientales, como el mantenimiento del paisaje o la lucha contra la erosión, o sociales, como la conservación del patrimonio cultural. Muchas de estas funciones no son comercializables. A la capacidad de los sistemas agroalimentarios locales para generar una amplia variedad de output se denomina "multifuncionalidad".

El presente trabajo parte de las premisas teóricas ofrecidas por los enfoques normativos sobre la multifuncionalidad de los espacios rurales, que se basan en la idea de que la eficiencia económica no ha de ser el único parámetro a optimizar mediante las políticas agrarias y de desarrollo rural (Wilson, 2009). La multifuncionalidad de los espacios rurales depende de diversos tipos de interacciones, como las existentes entre los bienes comerciales y los bienes públicos, o bien entre las dinámicas socioeconómicas y culturales y los procesos evolutivos del medio biofísico.

En el caso específico de las denominaciones de origen protegidas (DOP), así como de otros signos distintivos de calidad diferencial con vinculación al desarrollo territorial, el objetivo de los agentes económicos es recrear el mercado de determinados bienes públicos a través del etiquetado, lo que permitiría a los consumidores mostrar su disposición a pagar por determinados atributos inherentes a un bien comercial como el aceite de oliva. Sin embargo, las DOP de aceite de oliva españolas no obtienen aún en la actualidad rentas de diferenciación suficientes asociadas a la etiqueta de calidad diferencial. Sin embargo, la acción colectiva derivada de la organización interprofesional que tiene lugar en el ámbito de las DOP puede potenciar la generación de bienes públicos.

Frente al hecho de ser proveedores a la sociedad de bienes públicos y, por el contrario, no internalizar suficientemente las externalidades derivadas de su actividad en forma de rentas de diferenciación, las políticas públicas pueden promover, mediante sus sistemas de incentivos, que los sistemas agroalimentarios locales $(\mathrm{SIAL})^{2}$ cumplan con otras funciones sociales y ambientales demandadas por el conjunto de la sociedad.

En el ámbito del análisis de los estudios normativos de valoración de la demanda de ex-

2. El concepto de SIAL, como objeto de estudio, corresponde a una concentración espacial de explotaciones agrarias, empresas e instituciones en red, especializadas en un sector agroalimentario y que tienen en común toda una serie de activos específicos vinculados a un territorio, lo que ofrece una gran diversidad de respuestas, tanto desde el ángulo de los factores que determinan la tipicidad del producto como del relativo a sus efectos en el desarrollo local y rural (Muchnik et al., 2008; Sanz-Cañada y Muchnik, 2011). 
ternalidades, proponemos un proceso de toma de decisiones que emplea una metodología de identificación y valoración multicriterio, sobre la base de las preferencias reveladas por expertos, de las externalidades positivas territoriales asociadas a las DOP de aceite de oliva. Este modelo de valoración, que utiliza técnicas de Proceso Analítico de Redes (ANP), incorpora a los análisis de demanda de externalidades agroambientales otros bienes públicos, económicos y socioculturales con un enfoque de carácter territorial. Hasta la fecha, la investigación en la estimación de funciones de bienestar social agroalimentarias ha abordado escasamente la valoración de las externalidades territoriales, centrándose mayoritariamente en cuestiones relativas a las externalidades ambientales de la agricultura. Este trabajo contribuye a la literatura científica aportando un nuevo método para afrontar problemas de decisión multicriterio que permite considerar variables intangibles.

Los agrosistemas de olivar constituyen un ejemplo de gran interés a la hora de analizar el comportamiento multifuncional de los sistemas agrarios, pues se trata de un sector que tiene importantes implicaciones desde el punto de vista de la superficie ocupada (más de 2,5 millones de hectáreas en España), de la gestión del paisaje, del patrimonio natural y del medio ambiente. Por una parte, determinadas externalidades, como es el caso de la erosión y la degradación de los suelos, están convirtiéndose en un problema ambiental de primer orden (Sanz-Cañada et al., 2012), sobre todo en el olivar de montaña, que ocupa más de 800.000 ha en España. Asimismo, el olivar español ha experimentado hasta épocas recientes fuertes procesos de intensificación, impulsados por una Política Agrícola común (PAC) que tuvo hasta la campaña 2003/04 un corte marcadamente productivista, así como por la gran influencia de las casas comerciales de venta de insumos en las prácticas culturales de los agricultores. Sin embargo, el olivar es un cultivo en el que los paisajes de mayor valor ambiental y cultural, constituidos por olivares tradicionales, se están viendo progresivamente abocados a una situación de marginalización económica, debido a su escasa competitividad con respecto al olivar intensivo y a la situación estructural de bajos precios en los mercados internacionales del aceite.

Por otra parte, las DOP de aceite de oliva también constituyen un ejemplo de gran interés no sólo a la hora de analizar el comportamiento multifuncional de los SIAL, sino también de las posibilidades de contribución de dichas instituciones a la gobernanza de los territorios. Pueden constituir fórmulas de organización institucional e interprofesional de los alimentos identitarios. Los beneficios potenciales de las DOP no se encuentran relacionados tan sólo con la obtención de dichas rentas de diferenciación del bien comercial, sino también con su capacidad de contribuir positivamente a la gobernanza territorial en su conjunto. Otras instituciones de desarrollo oleícola local pueden también contribuir favorablemente a la gobernanza local. Las redes de colaboración que se construyen entre diferentes instituciones y agentes locales pueden promover, como resultado de la acción colectiva, procesos de gobernanza territorial que trascienden el objetivo originario de agregar valor al producto, en distintos ámbitos de actuación (Barjolle et al., 2007; PerrierCornet y Sylvander, 2000; Sanz-Cañada y Macías-Vázquez, 2005).

Los objetivos del trabajo son, en primer lugar, identificar las externalidades territoriales positivas inducidas por los sistemas oleícolas locales. En segundo término, se pretende determinar su valor relativo a partir de las preferencias reveladas por los expertos. Además, la relación entre la producción y la generación de externalidades depende del tipo de sistema de cultivo que se implante. En consecuencia, un tercer objetivo del trabajo es determinar las prefe- 
rencias de los expertos locales con respecto al fomento de los distintos sistemas de cultivo (tradicional, de producción integrada, ecológico o que se abandone), a partir de la respectiva provisión de externalidades territoriales que ofrecen cada uno de ellos.

Se parte de la hipótesis de que la rentabilidad socioeconómica, cultural y ambiental de los sistemas oleícolas locales es superior a la rentabilidad privada, dado que la sociedad no tiene en cuenta estas externalidades positivas. Por tanto, cabría considerar la propuesta de mecanismos públicos de compensación a los productores frente a estos fallos del mercado. En definitiva, se trata de aportar argumentos que permitan justificar y diseñar una política sobre bienes públicos en los sistemas oleícolas locales, mediante la retribución de las externalidades positivas que generan.

La elección de las DOP de Sierra de Segura (Jaén) y de Estepa (Sevilla y Córdoba) se ha realizado en función de los siguientes criterios: i) en ambos casos tienen una tradición organizativa, necesaria a la hora de valorar los procesos de gobernanza territorial, tanto en lo que concierne a la existencia de experiencias de comercialización de segundo grado como de la puesta en marcha de iniciativas de producción integrada (Estepa) y de agricultura ecológica (Sierra de Segura); ii) contemplan agrosistemas de olivar con diferentes tipologías -montaña, colina y campiña-.

\section{Marco teórico}

El concepto de multifuncionalidad de la agricultura se generaliza en la Conferencia de las Naciones Unidas sobre Medio Ambiente y Desarrollo celebrada en Río de Janeiro en 1992 (Cumbre de Río) ${ }^{3}$. A partir de entonces, su uso se difunde en los foros internacionales, primero en un contexto comercial (Celebración de la Ronda de Uruguay en 1994; Celebración del cincuenta aniversario de la creación de la FAO-Declaración de Quebec, 1995) y más tarde con un carácter político (Declaración de Ministros de Agricultura y del Consejo Europeo en 1997; Declaración de Ministros de la OCDE en 1998).

La Unión Europea (UE), ante la necesidad de abandonar la senda de la producción de commodities y de buscar una solución en las conversaciones de la OMC, impulsa el enfoque normativo de la multifuncionalidad rural para la reforma de la Política Agrícola Común (PAC) en 1992: este concepto se consolida con la Declaración de Cork (1996) y con la denominada Agenda 2000. A partir de entonces, tiene lugar un punto de inflexión en las políticas agrarias de la UE, marcado por un desplazamiento paulatino del peso de la intervención pública desde las políticas de mercados agrarios hacia las de desarrollo rural (el segundo pilar de la PAC), en busca de estrategias de diversificación económica, sostenibilidad en la producción agroalimentaria y multifuncionalidad de los espacios rurales. El principio de la multifuncionalidad se encuentra plenamente integrado en el actual Reglamento Europeo de Desarrollo Rural, de comienzos de 2007, y va a tener un papel fundamental en la reforma de la PAC para el periodo 2014-2020.

Muchos de los beneficios derivados de la multifuncionalidad se derivan de que las producciones agroalimentarias locales, así como los espacios rurales en su conjunto, generan externalidades o bienes públicos: al no existir mercado para ellos, los agentes económicos no obtienen remuneración alguna por la producción de externalidades, que a su vez no son consideradas en sus procesos de toma

3. Para profundizar en este concepto, véanse Gómez-Limón y Barreiro (2007) y OECD (2008). 
de decisiones. Por tanto, si se quiere que los SIAL desempeñen otras funciones diferentes a las estrictamente productivas, cabe pensar en la intervención del sector público ${ }^{4}$. Sin embargo, el hecho de que la producción de múltiples output se lleva a cabo de forma conjunta plantea el problema de aplicar medidas diferentes para cada uno de los objetivos de política agraria. En concreto, en el esbozo de las medidas encaminadas a la internalización de estas externalidades se plantean diversos problemas: la estimación correcta de su valor social, el carácter territorial que les caracteriza, los costes relacionados con la recogida de la información o el diseño de las políticas y las medidas legales necesarias para llevarlas a cabo (Reig, 2002).

Efectivamente, existe un interés creciente en la reorientación de las intervenciones públicas en el sector agrario y en la búsqueda de nuevos instrumentos que contribuyan no sólo a la sostenibilidad económica, sino también a la sostenibilidad ambiental y social de las zonas rurales. En este sentido, ya hace tiempo que la Comisión Europea señala como funciones de la agricultura europea, además de la productiva, la conservación del medio ambiente y del paisaje rural y la contribución a la viabilidad de las áreas rurales y a un desarrollo territorial equilibrado (Atance y Tió, 2000).

Determinadas contribuciones bibliográficas recientes, vinculadas a los enfoques de carácter normativo, coinciden en señalar que ninguna modelización de carácter disciplinar puede llegar a comprender y a valorar los diferentes tipos de interacciones que tienen lugar al abordar el problema de la multifuncionalidad de los espacios rurales (Cairol et al., 2009; Mann y Wüstemann, 2008; Renting et al., 2009; Rossing et al., 2007; Stobbelaar et al., 2009; Zander et al., 2007). Sin embargo, el principal problema que subsiste en estos enfoques es la necesidad de crear nuevas aproximaciones metodológicas y mucha mayor investigación empírica que contemple la alta diversidad de comportamientos multifuncionales que tienen los SIAL.

En el exterior de la UE, quienes también optan por esquemas de multifuncionalidad agraria (Noruega, Japón, Suiza y Corea) lo hacen, esencialmente, por sus efectos positivos sobre la seguridad en el abastecimiento de productos agroalimentarios o bien sobre el desarroIlo rural y la protección del medio ambiente (Potter y Burney, 2002). Por ejemplo, una política de transferencias a los productores agrarios podría inducirles a suministrar ciertas cantidades de bienes ambientales, como podría ser la absorción de $\mathrm{CO}_{2}$, con objeto de frenar el cambio climático (Hartell, 2004). También es probable que las políticas que conducen a la reducción del consumo de fertilizantes y al uso de abonos orgánicos conlleven un descenso en las emisiones de $\mathrm{N}_{2} \mathrm{O}$. Normalmente, este tipo de pagos requiere que los agricultores adopten ciertas prácticas que contribuyan al logro de los resultados medioambientales que se pretenden: entre otras, la extensificación de cultivos o la utilización de técnicas que limiten la erosión del suelo y la contaminación difusa. Además, los resultados alcanzados en términos de obtención de servicios medioambientales dependen del tipo de cultivo y de su localización. Una segunda justificación, más polémica, descansa en la precaria situación de algunas tierras marginales, dependientes de las ayudas económicas para poder subsistir y a las cuales la liberalización comercial podría impulsar al abandono de la producción.

El estudio de las funciones que desarrolla el cultivo del olivar se ha abordado desde diferentes aproximaciones metodológicas: expe-

4. La intervención del sector público tampoco garantiza que se alcance la eficiencia en el caso de los bienes públicos; y no sería necesaria si las externalidades son irrelevantes desde el punto de vista económico. 
rimentos de elección (vinculado a los enfoques de carácter positivo sobre la multifuncionalidad) y técnicas de decisión multicriterio tales como el Proceso Analítico Jerárquico (AHP, en sus siglas en inglés) y su generalización, el Proceso Analítico de Red (que se enmarcan en el ámbito de los enfoques normativos). Casi todos los trabajos han utilizado el olivar andaluz como ámbito de aplicación. Así, para estimar la demanda social de los atributos del olivar, Kallas et al. (2006) y Arriaza et al. (2008) utilizan los experimentos de elección. La técnica del AHP permite a Parra et al. $(2005,2008)$ analizar las funciones del olivar (económicas, técnicas, socioculturales y medioambientales) según el tipo de sistema de cultivo (convencional, integrado o ecológico). Un análisis detallado de las funciones medioambientales, segmentado en función de los distintos sistemas de cultivo, puede verse en Parra et al. (2007). Gómez-Limón y Riesgo (2012) desarrollan una metodología para el análisis empírico de la sostenibilidad de las explotaciones de olivar en Andalucía, en aspectos próximos al análisis de la multifuncionalidad, que cumple las siguientes condiciones: abarcar la triple dimensión del concepto (económica, social y ambiental) y, a nivel operativo, basarse en un conjunto de indicadores que posibilite un diagnóstico preciso. Fuera de España, con una metodología diferente, Fleskens et al. (2009) evalúan el papel que las múltiples funciones de los agro-ecosistemas olivareros desempeñan en el proceso de toma de decisiones, aplicándolo al olivar de montaña del nordeste de Portugal. Estos trabajos concluyen, entre muchos otros aspectos, en que la relación entre producción y generación de externalidades depende del tipo de sistemas de cultivo y de las prácticas agrarias y agroambientales que se lleven a cabo.

En la literatura internacional, el ANP se ha utilizado en el análisis de la multifuncionalidad agraria en Holanda (Parra et al., 2008b) y en otras áreas de conocimiento muy diversas (Köne y Büke, 2007; Chang et al., 2007;
Yuksell y Dagdeviren, 2007; Khan y Faisal, 2008; Wolfslehner y Vacik, 2008 o Promentilla et al., 2008, entre otros). En España, esta técnica se ha utilizado para la valoración de tierras agrícolas (García-Melón et al., 2008), el uso de suelo industrial (Aragonés-Beltrán et al., 2008), la evaluación del riesgo de erosión en el olivar de montaña (Nekhay et al., 2009), la sostenibilidad del cultivo del arroz (Reig et al., 2010), los inmuebles urbanos (Aznar et al., 2010) o los resultados universitarios (Cortés-Aldana et al., 2010). En el ámbito oleícola, Arriaza y Nekhay (2010) combinan las técnicas de análisis del AHP y del ANP en el olivar andaluz de baja producción. EI ANP les sirve a Carmona et al. (2011) para estudiar el impacto de diversas prácticas agrarias (variedad de aceituna, gestión del suelo, sistema de riego, utilización de fertilizantes y fitosanitarios, momento y forma en que se cosecha, entre otros) en el olivar andaluz.

\section{Material y métodos}

El ANP fue propuesto por Saaty $(2001,2005)$ y representa un problema de decisión como una red de criterios y alternativas llamados elementos y agrupados en clusters. Cada elemento puede tener influencia sobre el resto de elementos de la red, lo que significa que una red puede incorporar interdependencia entre elementos de un mismo cluster (dependencia interna) o interdependencia entre elementos de clusters diferentes (dependencia externa). Esta característica del ANP permite trabajar con criterios y alternativas interdependientes y proporciona un enfoque preciso para modelizar entornos reales complejos. Una vez construido el modelo en red, el ANP comprende los siguientes pasos (Meade y Sarkis, 1999; Saaty, 2005; Lee et al, 2009).

- Identificación de los elementos relevantes: criterios y alternativas; y formación de los 
clusters agrupando dichos criterios por similitud. Las alternativas constituyen un último cluster.

- Elaboración de una matriz de influencias donde las filas $i$ y las columnas $j$ son los criterios, de tal manera que los elementos de la matriz $n_{i j}$ toman valor 1 cuando el criterio de la fila influye en el criterio de la columna y 0 , en caso contrario. Habitualmente, estas influencias son determinadas por el decisor que, en nuestro caso, está constituido por expertos conocedores de la realidad objeto de estudio, para quienes no es difícil definir qué criterios influyen sobre cada uno del conjunto de criterios que se consideren. Esta matriz se necesita para diseñar la red; es decir, para establecer las influencias internas y externas entre los elementos.

- Elaboración de un cuestionario que incluya todas las preguntas que van a hacerse a los expertos consultados. Dos criterios se comparan al mismo tiempo con respecto a un tercero que actúa como criterio de control. Es decir, el decisor se enfrenta a comparaciones por pares de criterios que influyen en un tercero y únicamente deben señalar qué criterio de los dos (y hasta qué punto) influye más en ese tercer criterio de control. La valoración de la intensidad de la preferencia se lleva a cabo utilizando la escala fundamental de Saaty (2001), cuyo significado es: 1, "ambos criterios son igualmente importantes"; 3, "un criterio es ligeramente más importante que el otro"; 5, "un criterio es bastante más importante que el otro"; 7, "un criterio es mucho más importante que el otro"; y 9, "un criterio es extremadamente más importante que el otro". Las respuestas agregadas (calculando medias geométricas) son los inputs que utiliza Superdecisions ${ }^{5}$ para calcular la que se denomina unweighted super matrix. Ésta es una matriz cuyos elementos tienen el significado de en qué medida los criterios y alternativas de las filas influyen en los que aparecen en las columnas. El programa calcula, además, una ratio de consistencia, que debe ser menor que 0,1 para asegurar la consistencia de las respuestas de los entrevistados.

- Esta primera matriz no es estocástica, por lo que se calcula una segunda matriz ponderando los elementos de la primera con los pesos que los decisores han dado a las influencias que los clusters tienen entre sí. Se obtiene así una matriz estocástica por columnas (weighted super matrix).

- Elevación de la matriz ponderada a potencias sucesivas, hasta alcanzar la convergencia con la limit super matrix. Todos los valores obtenidos en esta matriz limit suman 1 y el valor correspondiente a cada elemento (criterios y alternativas) indica la influencia que cada elemento tiene en el conjunto del sistema. Si se vuelven a normalizar entre sí los valores correspondientes a las alternativas, entonces se obtiene la síntesis sólo para las alternativas.

La valoración de los clusters y los criterios responde a factores subjetivos de los expertos consultados, por lo que la selección que se haga de éstos es un elemento crucial del análisis para evitar sesgos. En nuestro caso, se trató de consultar a un número relativamente amplio de personas. Por una parte, se intentó buscar un equilibrio entre expertos locales, buenos conocedores del terreno y la realidad objeto de estudio y, por otra, investigadores en ciencias sociales y con experiencia reconocida en los ámbitos de los sistemas del olivar, la multifuncionalidad agraria y el desarrollo sostenible. Adicionalmente, se pre-

5. Software for Decision Making with Dependance and Feedback (SuperDecisions), desarrollado por Thomas L. Saaty y accesible en: http://www.superdecisions.com/ 
tendió encontrar un cierto equilibrio cuantitativo entre los entrevistados, en función de su respectiva sensibilidad o proximidad hacia las cuestiones económicas, ambientales y socio-culturales.

Como resultado, en el verano-otoño de 2011 se entrevistó a un total de 16 expertos locales en la DOP de Estepa y a 11 en la DOP de Sierra de Segura. Los mencionados expertos locales corresponden a: representantes de los Consejos Reguladores de las DOP, de las Oficinas Comarcales Agrarias, de los organizaciones profesionales agrarias, de las asociaciones de consumidores y de productores ecológicos y de los Grupos de Desarrollo Rural; gerentes de las comercializadoras de segundo grado; gerentes de determinadas cooperativas y almazaras privadas que llevan a cabo estrategias de diferenciación del aceite; técnicos de ATRIAs y de APIs. Mientras que los expertos locales contestaron a la primera parte del cuestionario -comparación de criterios-, 8 investigadores en ciencias sociales diseñaron la matriz de influencias y contestaron la segunda parte-comparación de alternativas y de clusters-, de contenido mucho más teórico: fue cumplimentada por investigadores del CSIC y del CITA de Aragón, por expertos del sector de la Junta de Andalucía y por profesores de las Universidades de Sevilla, Jaén y Zaragoza.

La selección de los diez criterios incluidos en el modelo ANP, que en su mayoría corresponden a externalidades territoriales pero que en algunos casos se refieren a funciones comerciales, ha sido realizada a partir de la bibliografía existente, de la información primaria obtenida mediante entrevistas a testigos cualificados y expertos y del debate entre los miembros del equipo de investigación. Se ha hecho un especial esfuerzo en reducir los criterios potenciales a diez, a fin de que el número de combinaciones de criterios no sea muy elevado y se pueda lograr que la respuesta al cuestionario sea factible. A conti- nuación, los criterios se han agrupado en tres grupos o clusters: económicos, ambientales y socio-culturales. Todos los criterios son cualitativos y se pide a los expertos que los valoren cuantitativamente a partir de la escala fundamental de Saaty en cinco niveles.

El primer cluster (1), el económico, se compone de cuatro criterios principales: la generación de rentas; la calidad diferencial; la acción colectiva en materia de comercialización; y la seguridad alimentaria.

- (1.1) La generación de rentas corresponde a las rentas relacionadas directamente con el cultivo del olivar y la producción del aceite de oliva, es decir, del bien comercial. En nuestro caso de las DOP, hacemos especial referencia a la obtención de rentas de diferenciación asociadas a la comercialización de aceite con etiqueta de calidad.

- (1.2) La calidad diferencial es el principal objetivo de la actividad de las DOP, lo que implica: i) la tipicidad del aceite, que es función de la composición varietal de los olivares locales; ii) un alto grado de excelencia en los aceites, que han de someterse a los requisitos del reglamento de la DOP y a la inspección de la entidad certificadora. Ello implica que los Consejos Reguladores desplieguen una importante actividad interprofesional basada en la difusión y el fomento de las prácticas críticas en la cadena de la calidad, tanto a los olivicultores como a las almazaras y a las empresas comercializadoras.

- (1.3) La existencia de iniciativas colectivas en materia de comercialización conjunta de los aceites locales bajo una marca común, hace referencia a la implantación de empresas comercializadoras de segundo grado, así como a otro tipo de acciones conjuntas en materia de marketing, como es la promoción colectiva de los aceites locales.

- (1.4) La seguridad alimentaria garantiza la inocuidad del aceite de oliva para la sa- 
lud del consumidor en términos de ausencia de residuos contaminantes. Aparece reflejada como un criterio económico para los agentes locales por el hecho de que cada vez más va a constituir un factor de competitividad para los SIAL oleícolas, mientras que podría ser considerada también como un criterio socio-cultural para el conjunto de la sociedad.

Respecto al segundo cluster (2), el ambiental, se compone de tres elementos: la lucha contra la erosión y la degradación de los suelos; la reducción de la contaminación difusa; y la conservación de la biodiversidad y el mantenimiento de los paisajes de olivar.

- (2.1) La lucha contra la erosión y la degradación de los suelos, que se combate mediante la implantación de cubiertas vegetales, se dirige a la minimización de su degradación física y química (por compactación, salinización, acidificación...) y de la pérdida del suelo por lluvia, escorrentía, viento y laboreo.

- (2.2) La reducción de la contaminación difusa en suelos y acuíferos se refiere a la minimización de la contaminación causada por sustancias contaminantes (fertilizantes, pesticidas, herbicidas, ...), que llegan a masas de agua como resultado de la lluvia, de la infiltración a través de los suelos o de la escorrentía superficial y por percolación a aguas subterráneas.

- (2.3) La conservación de la biodiversidad consiste en la preservación de un número de especies considerado óptimo, es decir, de la riqueza de especies biológicas, que es variable en el tiempo y está referida a un espacio arbitrariamente definido y representativo de un ecosistema. Con respecto al mantenimiento y mejora de los paisajes de olivar, incidimos en la conservación y el fomento de un mosaico de usos y de cobertura vegetal cuya finalidad sea mantener la variabilidad del paisaje y su multifuncionalidad.
Por último, respecto al cluster (3) de criterios socio-culturales, se toman en cuenta: la fijación de la población rural; el fomento de las relaciones entre instituciones y empresas locales y la creación de un entorno innovador; y las actividades de puesta en valor del patrimonio cultural y natural.

- (3.1) En cuanto a la fijación de la población rural, el cultivo del olivar forma parte de un conjunto de rentas y de empleos complementarios para las familias rurales, sobre todo en el caso de las zonas de monocultivo de olivar, como es el caso de las DOP objeto de estudio. La generación de empleo y de rentas en los SIAL oleícolas, tanto directos como indirectos, resulta un elemento central del esquema de rentas y de ocupación laboral de las poblaciones locales, posibilitando el dinamismo de las comunidades rurales (propietarios, cooperativas, distribuidores...) y, por tanto, frenando la despoblación rural.

- (3.2) El fomento de las relaciones entre instituciones y empresas locales y la creación de un entorno innovador son condiciones necesarias para la existencia de relaciones de gobernanza territorial. La adopción de innovaciones y conocimientos constituye un objetivo de carácter instrumental para la consecución de un cierto grado de gobernanza ambiental (por ejemplo, el aprendizaje colectivo del manejo de la cubierta vegetal para combatir la erosión), para la obtención de un aceite de calidad diferencial, para la comercialización en común, así como para cualquier otro tipo de criterios económicos, socio-culturales o ambientales.

- (3.3) Las actividades de puesta en valor del patrimonio cultural y natural se traducen en el fomento de actividades de diversificación concéntrica de los SIAL oleícolas, tales como el turismo y las rutas oleícolas, la fabricación de jabones y cosmética o la artesanía con madera de olivo. 
Tanto los criterios socio-culturales como los ambientales hacen referencia a objetivos de retribución de las externalidades positivas. Por el contrario, entre los criterios económicos, dos de ellos corresponden a objetivos de mejora de las rentas del bien comercial, el aceite de oliva -"generación de rentas" y "acción colectiva en materia de comercialización"-, mientras que otros dos -"calidad diferencial" y "seguridad alimentaria"- tienen un objetivo mixto de obtención de rentas comerciales y de retribución de externalidades ${ }^{6}$.

Las alternativas de sistemas de cultivo que se proponen (cluster 4) son las siguientes: el cultivo ecológico, el cultivo de producción integrada, el cultivo convencional y el abandono del cultivo.

\section{Resultados}

Partiendo de las respuestas que los expertos de las dos zonas han dado en los cuestionarios, con el software Superdecisions se calcularon las matrices unweighted, weighted y limit para cada una de las zonas y se comprobó que las ratios de consistencia no superaban el 0,10 . A partir de la matriz limit, se obtuvieron los vectores de prioridades de las alternativas y de los criterios. En el Anexo pueden verse las tres tablas correspondientes a cada una de las dos zonas.

En relación con qué alternativa o sistema de cultivo proporciona mayor valor a la sociedad, la figura 1 muestra claramente que corresponde a las prácticas más respetuosas con el medio ambiente, como son los cultivos ecológico e integrado, si bien el peso que ob- tienen uno y otro difiere respectivamente en las DOP de Estepa y de Sierra de Segura. El cultivo integrado se ha generalizado en la DOP de Estepa, debido a la acción interprofesional llevada a cabo por la comercializadora de segundo grado Oleoestepa, mientras que es casi inexistente en la DOP de Sierra de Segura, lo que explica que los expertos estepeños valoren más dicha práctica -un peso del 35 por ciento frente al 24 por ciento, respectivamente-. Por el contrario, en la DOP de Estepa, donde predomina el olivar de campiña y de pendiente moderada, no existe olivicultura ecológica, mientras que en la DOP de Sierra de Segura, donde prevalece el olivar de montaña, de pendientes medias y altas, existe una larga tradición en agricultura ecológica por parte de una cooperativa pionera, Sierra de Génave, así como un movimiento asociativo en agricultura ecológica: esto determina que la valoración sobre la olivicultura ecológica sea significativamente superior en la Sierra de Segura -44 por ciento frente al 32 por ciento de Estepa-. Las alternativas de cultivo convencional y de abandono de la producción obtienen pesos mucho más reducidos en ambas DOP -entre el 14 y el 19 por ciento-.

En la figura 2 se presentan los pesos normalizados de los clusters; es decir, la influencia relativa que los expertos otorgan a cada uno de los grupos de objetivos. En conjunto, las funciones económicas del olivar resultan ser las más importantes: en torno al 60 por ciento. Las funciones ambientales y las socioculturales se reparten, casi a partes iguales, el 40 por ciento restante.

No obstante, descendiendo al análisis de los criterios individuales, destaca como primera

6. La finalidad de las empresas ha de ser conseguir que, en el futuro, el consumidor pague unas rentas de diferenciación por las etiquetas de las DOP que incorporen plenamente los atributos de calidad diferencial y de seguridad alimentaria. Sin embargo, mientras esto no suceda, ambos atributos pueden ser remunerados parcialmente mediante las políticas públicas. 


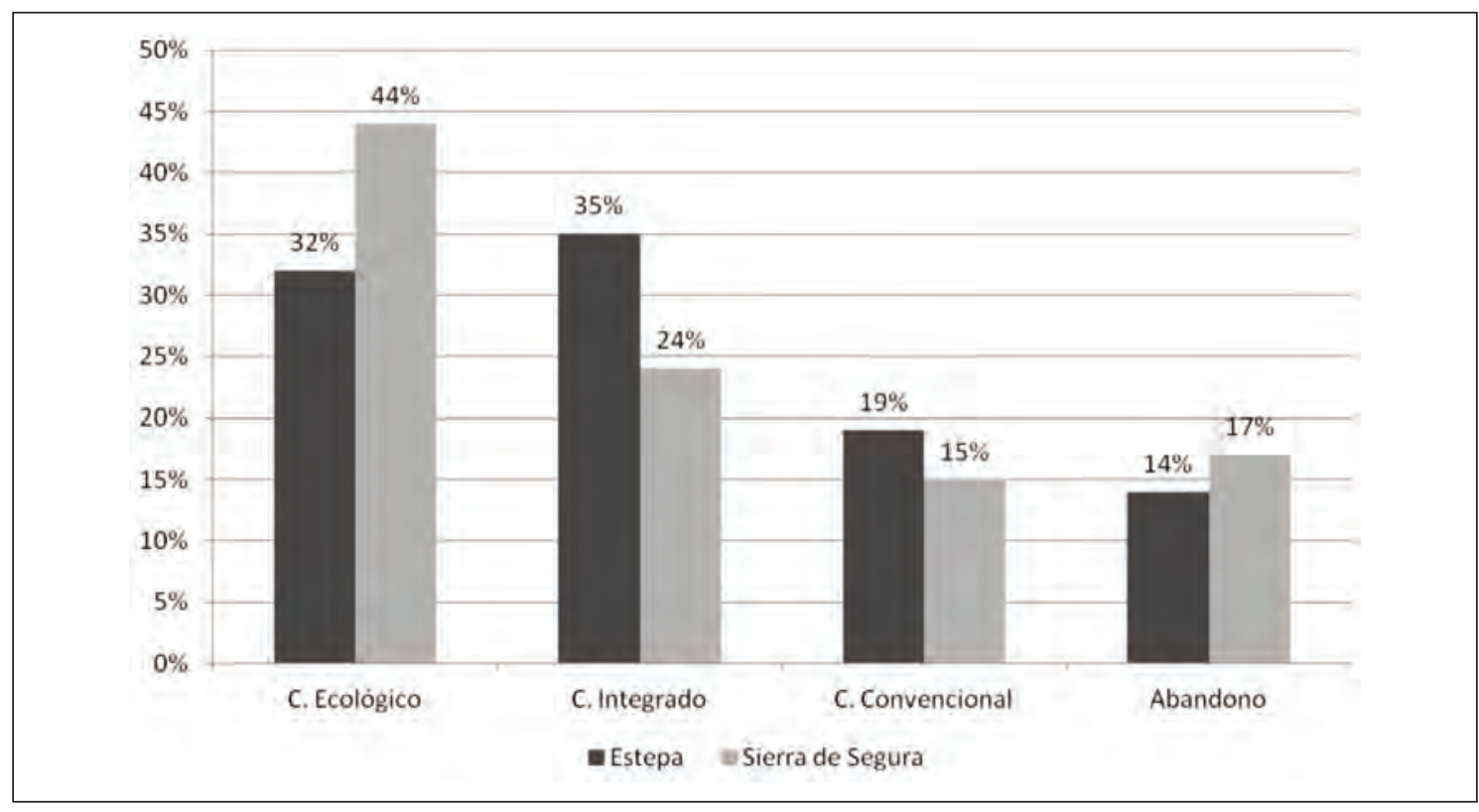

Figura 1. Prioridades de las alternativas de cultivo en las DOP de Estepa y de Sierra de Segura. Fuente: Elaboración propia.

Figure 1. Alternative crops priorities of the Protected Designations of Origin Estepa and Sierra de Segura.

Source: Own elaboration.

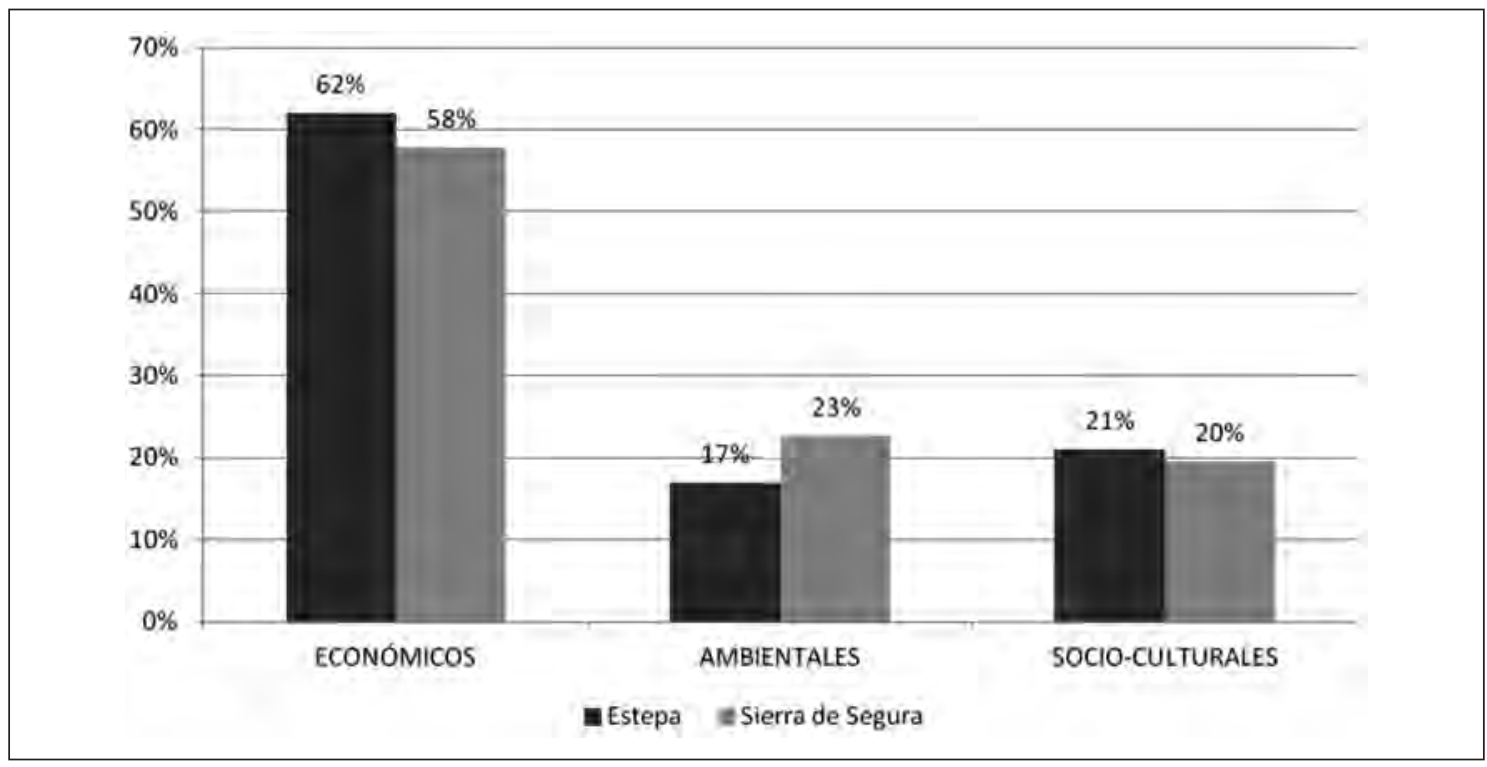

Figura 2. Prioridades de los clusters en las DOP de Estepa y de Sierra de Segura.

Fuente: Elaboración propia.

Figure 2. Clusters priorities of the Protected Designations of Origin Estepa and Sierra de Segura. Source: Own elaboration. 
función la 3.1 "Acción colectiva en materia de comercialización" (cuyo peso alcanza el 25 por ciento en Estepa y el 23 por ciento en Sierra de Segura), seguida de la 1.2 "Mejorar la calidad diferencial" (18 por ciento y 17 por ciento, respectivamente) y, en tercer lugar, la 3.2 "Creación de un entorno innovador" (17 y 16 por ciento). En la tabla 1 se reflejan estos resultados.
Es de destacar que, entre los criterios económicos, sobresalen, detrás del 1.3 "Acción colectiva en materia de comercialización" y del 1.2 "Mejora de la calidad diferencial", el 1.4 "Mejora de la seguridad alimentaria", quedando relegado al último puesto el 1.1 "Generación de rentas". Respecto a los criterios ambientales, el más importante es el 2.2 "Disminuir la contaminación difusa", se-

Tabla 1. Prioridades de los criterios en las DOP de Estepa y de Sierra de Segura

Table 1. Criteria priorities of the Protected Designations of Origin Estepa and Sierra de Segura

\begin{tabular}{lcc}
\hline & DOP Estepa & DOP Sierra de Segura \\
\hline ECONÓMICOS & $62,1 \%$ & $57,8 \%$ \\
\hline 1.1. Generación de rentas & $6,4 \%$ & $5,8 \%$ \\
1.2. Mejora de la calidad diferencial & $18,3 \%$ & $17,0 \%$ \\
1.3. Fomento de la acción colectiva en la comercialización & $25,1 \%$ & $22,7 \%$ \\
1.4. Mejora de la seguridad alimentaria & $12,4 \%$ & $12,2 \%$ \\
\hline AMBIENTALES & $16,9 \%$ & $22,6 \%$ \\
\hline 2.1. Lucha contra la erosión y degradación de los suelos & $7,1 \%$ & $9,9 \%$ \\
2.2. Disminución de la contaminación difusa & $8,4 \%$ & $10,8 \%$ \\
2.3. Conservación de la biodiversidad y de los paisajes de olivar & $1,4 \%$ & $1,9 \%$ \\
\hline SOCIO-CULTURALES & $21,0 \%$ & $19,6 \%$ \\
\hline 3.1. Fijación de la población rural & $1,7 \%$ & $1,6 \%$ \\
3.2. Creación de un entorno innovador & $17,2 \%$ & $15,9 \%$ \\
3.3. Puesta en valor del patrimonio natural y cultural & $2,2 \%$ & $2,2 \%$ \\
\hline
\end{tabular}

Fuente: Elaboración propia.

Source: Own elaboration.

guido muy de cerca por el 2.1 "Lucha contra la erosión y degradación de los suelos". Por último, entre los criterios socio-culturales, el más relevante es, de forma destacada, el 3.2 "Creación de un entorno innovador".
Para entender en qué medida los expertos creen que cada alternativa contribuye a generar cada una de las funciones, pueden verse las figuras 3 (DOP de Estepa) y 4 (DOP de Sierra de Segura). Se han elaborado a partir de los datos de las matrices unweighted. 


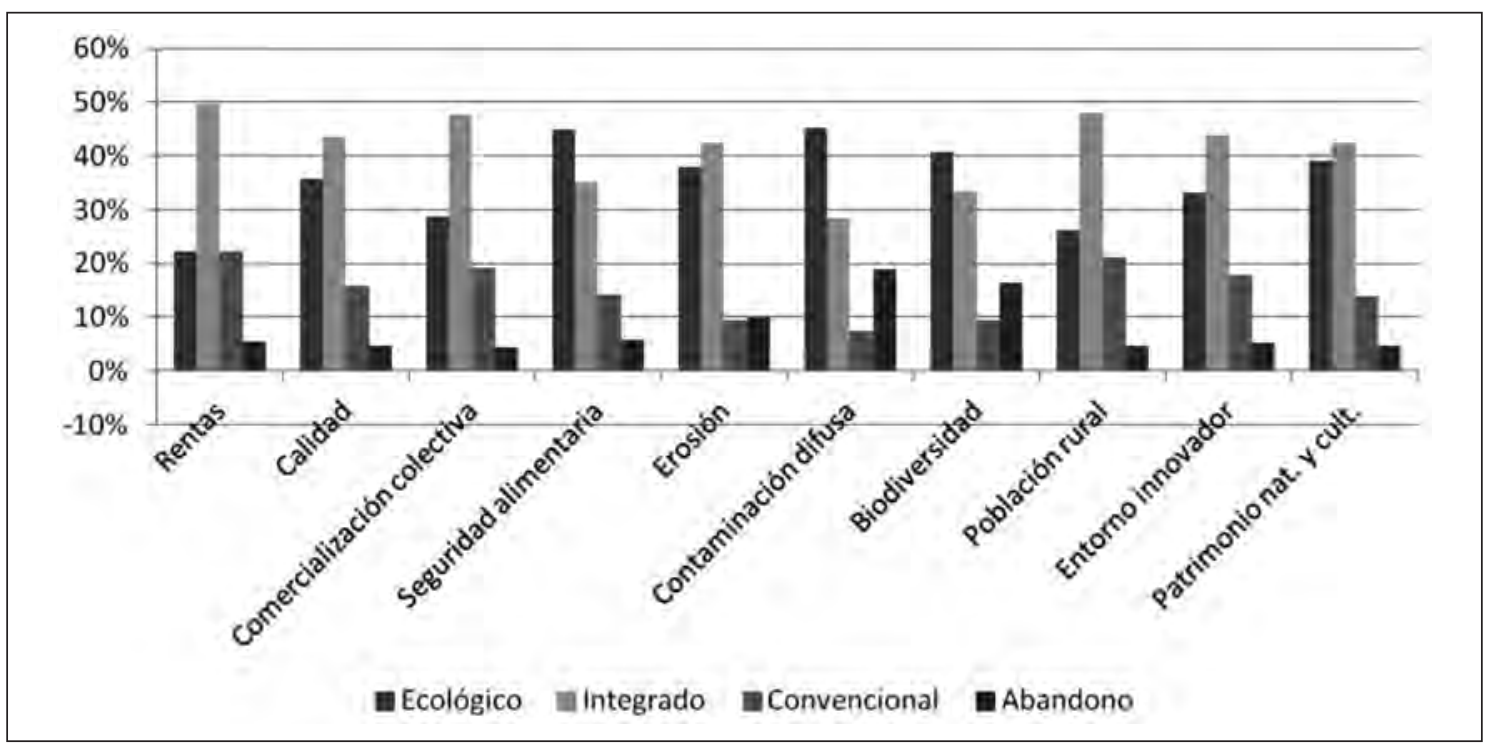

Figura 3. Influencia del tipo de cultivo en la consecución de las funciones. DOP de Estepa. Fuente: Elaboración propia.

Figure 3. Influence of the type of crop on the achievement of the functions in Protected Designation of Origin Estepa.

Source: Own elaboration.

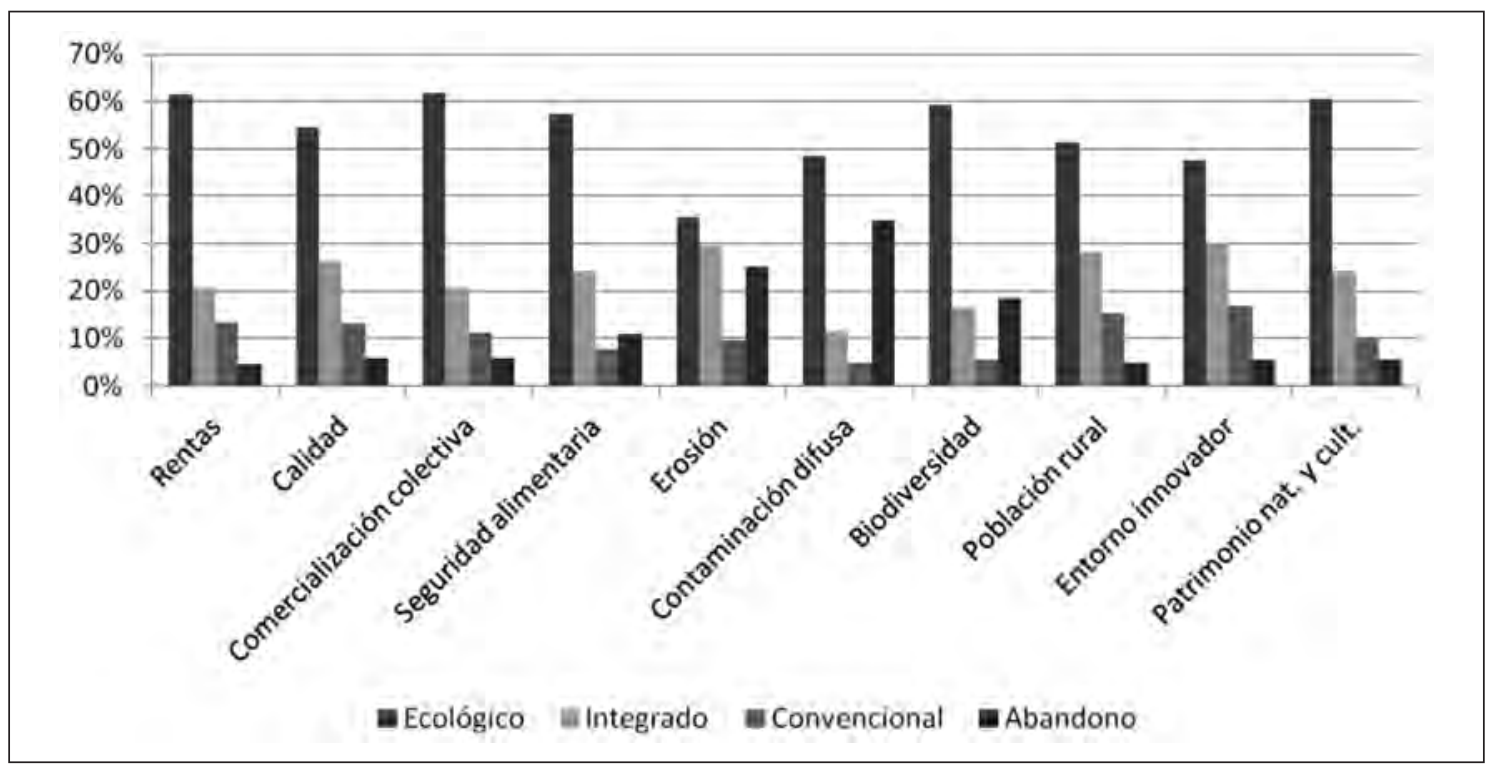

Figura 4. Influencia del tipo de cultivo en la consecución de las funciones. DOP Sierra de Segura. Fuente: Elaboración propia.

Figure 4. Influence of the type of crop on the achievement of the functions in Protected Designation of Origin Sierra de Segura.

Source: Own elaboration. 
En la DOP de Estepa, las respuestas dejan claro que los expertos opinan que el cultivo integrado es la mejor alternativa para la consecución de los objetivos económicos y socioculturales, pues el cultivo integrado acaba de generalizarse a la gran mayoría de la superficie olivícola. Únicamente se obtienen mayores logros en los criterios ambientales mediante el cultivo ecológico, salvo en el caso de la lucha contra la erosión.

Los resultados anteriores contrastan con la opinión de los expertos en la DOP de Sierra de Segura. En la figura 4 se aprecia rotundamente que el cultivo ecológico es el preferido para alcanzar todos los objetivos económicos, ambientales y socio-culturales.

Sin embargo, ambas DOP coinciden en juzgar al cultivo convencional y al abandono del cultivo como las alternativas significativamente peor valoradas.

\section{Discusión}

El estudio de la multifuncionalidad del olivar en las DOP de Estepa y de Sierra de Segura muestra que los expertos valoran preferentemente las externalidades y las rentas comerciales de carácter económico, aunque también consideran de forma emergente las externalidades ambientales y socio-culturales. Además, el tipo de sistema de cultivo determina una significativa variabilidad en las funciones de preferencia social en materia de multifuncionalidad. Mientras que en la DOP de Estepa se considera que la sostenibilidad ambiental, económica y socio-cultural tiende a maximizarse mediante el cultivo en producción integrada, en la DOP de Sierra de Segura se logra mediante el cultivo ecológico.

Los resultados de la aplicación del modelo ANP indican que los criterios que más pesan en la elección de la orientación productiva son fundamentalmente tres: la acción colec- tiva en materia de comercialización, la calidad diferencial y la creación de un entorno innovador. El elevado resultado que alcanzan los dos primeros criterios concuerda con los obtenidos por otros trabajos de investigación sobre preferencias valoradas mediante paneles de expertos (Sanz-Cañada et al., 2011): los problemas relativos al déficit de conocimiento de los consumidores sobre el aceite de oliva y al escaso desarrollo de las estrategias de comercialización aparecen destacados como los problemas que, desde el punto de vista del análisis vertical inherente al análisis de cadenas oleícolas en España, requieren ser resueltos con mayor urgencia. Asimismo, concluimos que las relaciones de gobernanza territorial centradas en la creación de un entorno innovador desempeñan un papel muy relevante en la generación de rentas comerciales y de las diferentes externalidades territoriales, ya que se erigen en variables instrumentales, expresadas en forma de condición necesaria, para la obtención de un nivel de logro adecuado en cualquier otro tipo de objetivos.

Por otra parte, dos criterios ambientales, como son la lucha contra la erosión y la reducción de la contaminación difusa, y un criterio económico, la seguridad alimentaria, alcanzan valores intermedios en nuestro modelo. Sin embargo, estos resultados, que son fruto de las opiniones de los expertos locales, obtienen una valoración claramente más baja que la ofrecida por los trabajos de investigación que consideran colectivos amplios de expertos a escala nacional, como es el caso de Sanz-Cañada et al. (2011): aquí, el alto grado de erosión y degradación de los suelos es calificado como el primer problema ambiental y territorial del olivar español, mientras que la contaminación difusa y la seguridad alimentaria también son juzgados como problemas bastante importantes. A la hora de definir la composición de los paneles de expertos a distintas escalas geográficas, la 
principal diferencia es que, a nivel local, determinados colectivos, como por ejemplo los ambientalistas, pueden llegar a escasear.

Por el contrario, la conservación de la biodiversidad y de los paisajes de olivar, así como la puesta en valor del patrimonio natural y cultural, aparecen todavía como criterios muy poco valorados por los expertos, quizás porque son todavía escasamente tomados en consideración incluso por buena parte de dicho colectivo. La generación de rentas y la fijación de la población rural también logran coeficientes de ponderación pequeños, sobre todo en el segundo caso, quizás por el hecho de ser variables de carácter finalista: la aplicación de las políticas sobre bienes públicos genera efectos positivos en dichos objetivos, pero de forma indirecta y en un plazo temporal más largo que en el caso de otros criterios.

Un mayor grado de análisis de los resultados obtenidos por la aplicación del ANP podría lograrse, cara a futuras investigaciones, mediante la aplicación de técnicas de grupos de discusión a cada uno de los colectivos de expertos de las DOP. La finalidad será elaborar "prioridades de consenso" entre todos los participantes y aportar información cualitativa para la explicación de los factores que potencian o limitan los esquemas locales de preferencias.

Por otra parte, la clasificación de los criterios examinados en un cluster determinado (económico, socio-cultural o ambiental) es, por definición, difusa. En consecuencia, futuros desarrollos del modelo pueden consistir en la realización de nuevas aplicaciones del ANP a diferentes re-clasificaciones de los criterios en nuevos clusters: por ejemplo, un cluster económico-social-cultural frente a otro cluster ambiental. La comparación de los resultados que se pudieran obtener con los ofrecidos en el presente trabajo nos permitiría avanzar en la definición de hipótesis sobre el grado de dependencia o independencia entre los diferentes tipos de clusters.

Los resultados del presente trabajo sirven para suministrar información a los decisores de las políticas agrarias y de desarrollo rural, ya que se obtiene un sistema de ponderación de la influencia relativa otorgada por los expertos a los diferentes criterios económicos, ambientales y socio-culturales, en materia de externalidades territoriales y de bienes comerciales.

\section{Agradecimientos}

Este estudio se enmarca en el proyecto CSO2009-08154 del Plan Nacional de I+D+i, "Externalidades territoriales en sistemas agroalimentarios locales: desarrollo rural, paisajes y bienes públicos en denominaciones de origen de aceite de oliva (EXTERSIAL)", cuyo investigador principal es Javier Sanz Cañada. Los autores desean también mostrar su agradecimiento a los agentes sociales entrevistados en las áreas de estudio y a los expertos científicos que también fueron consultados. Por último, las sugerencias de dos evaluadores anónimos han contribuido a mejorar el trabajo. 


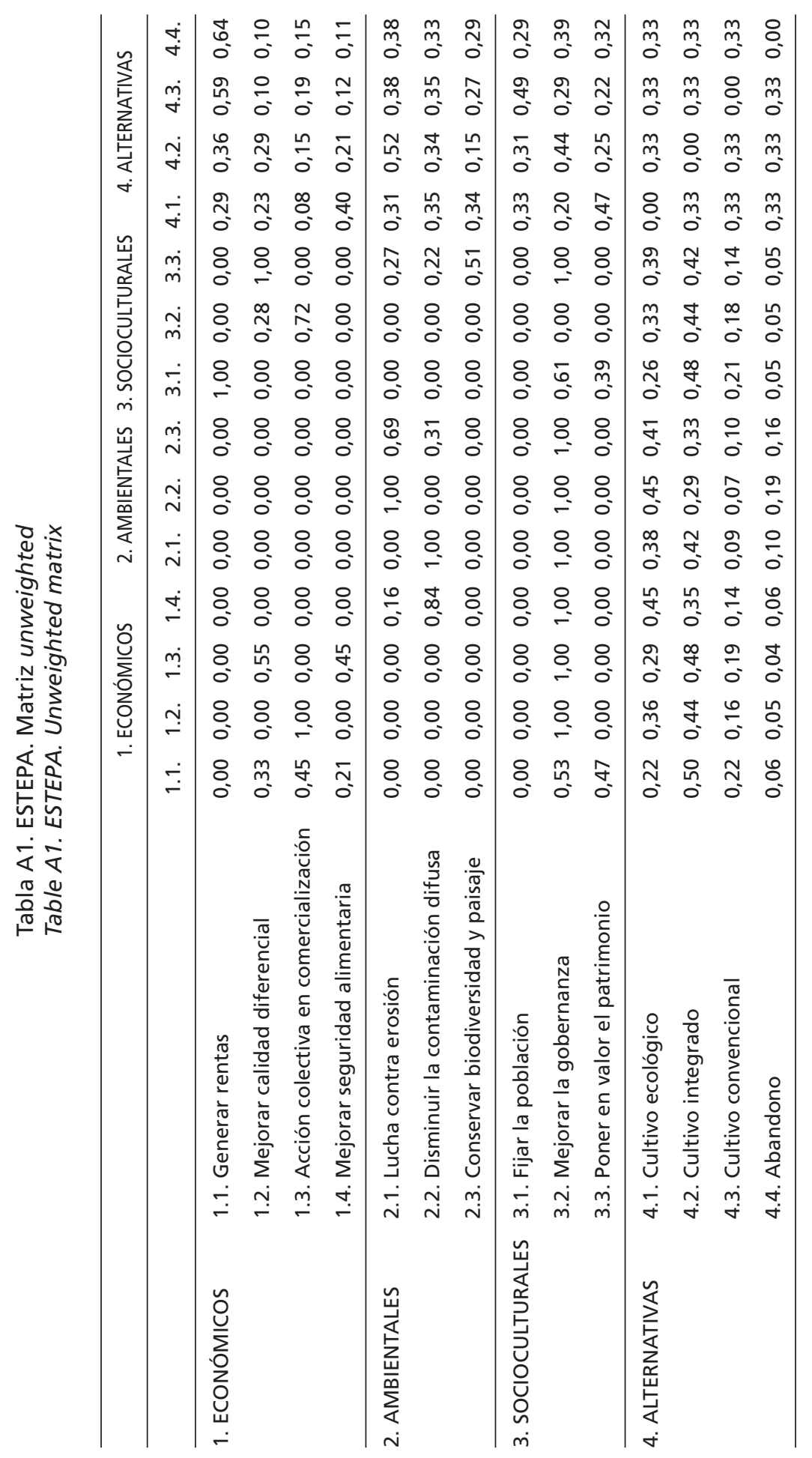




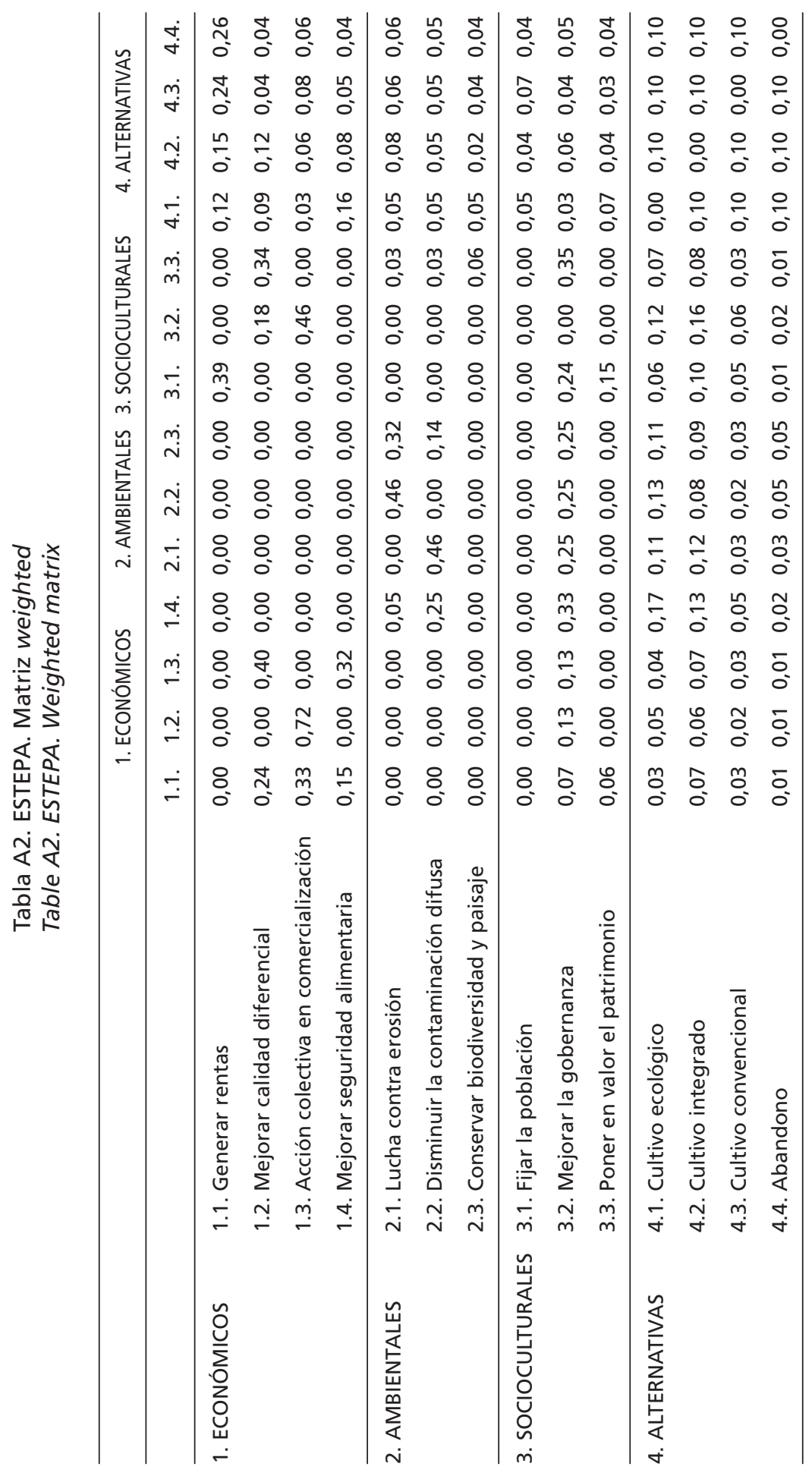




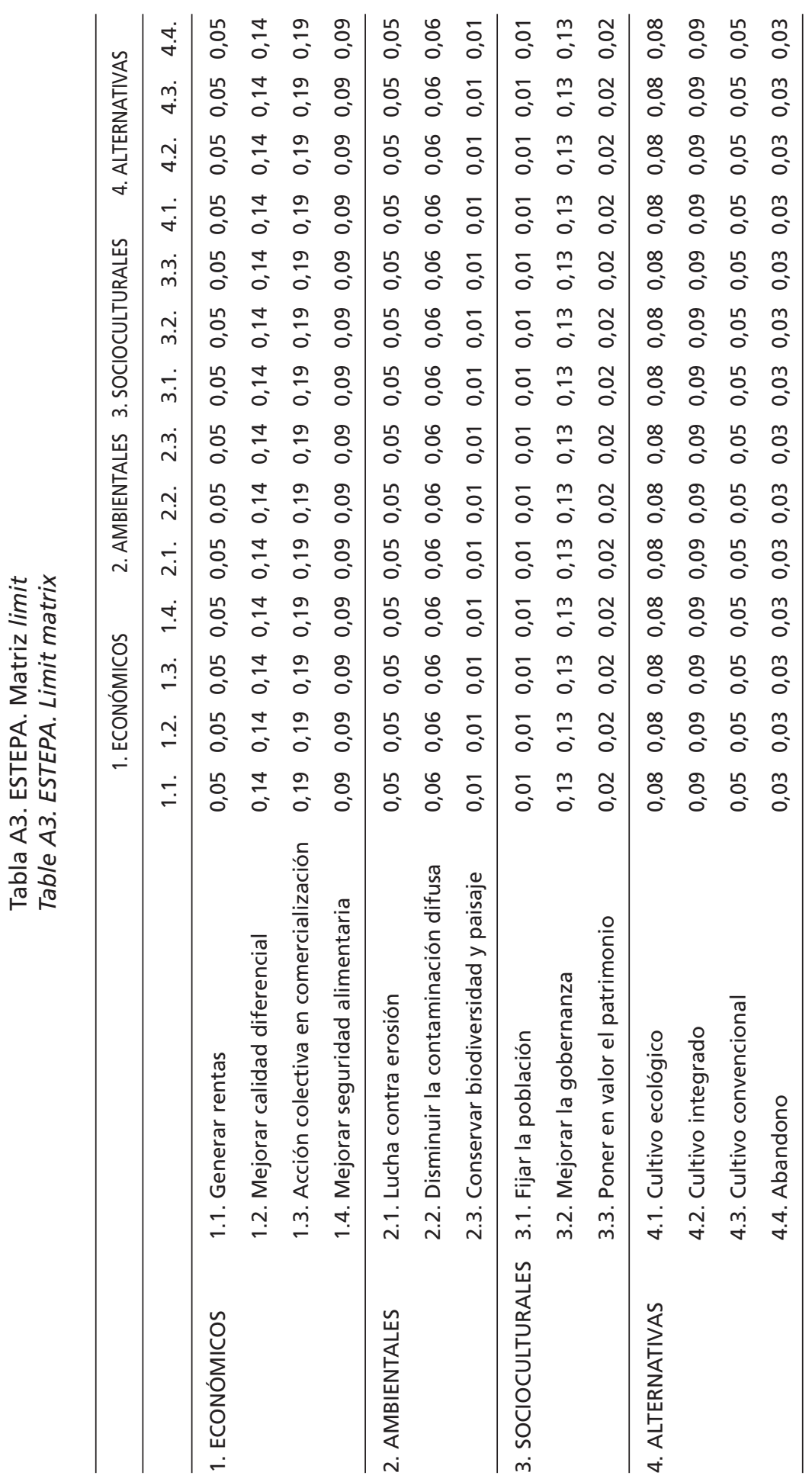




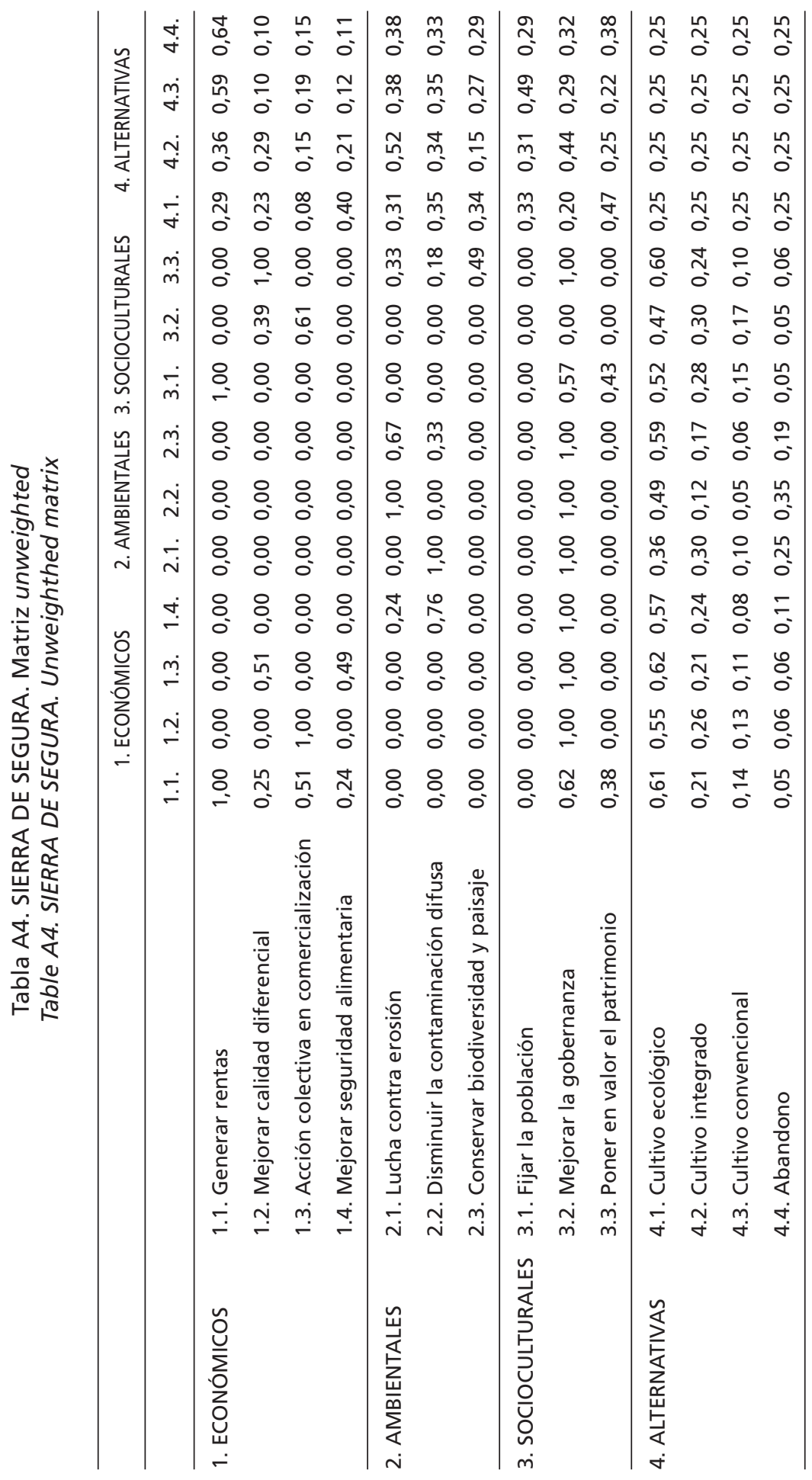




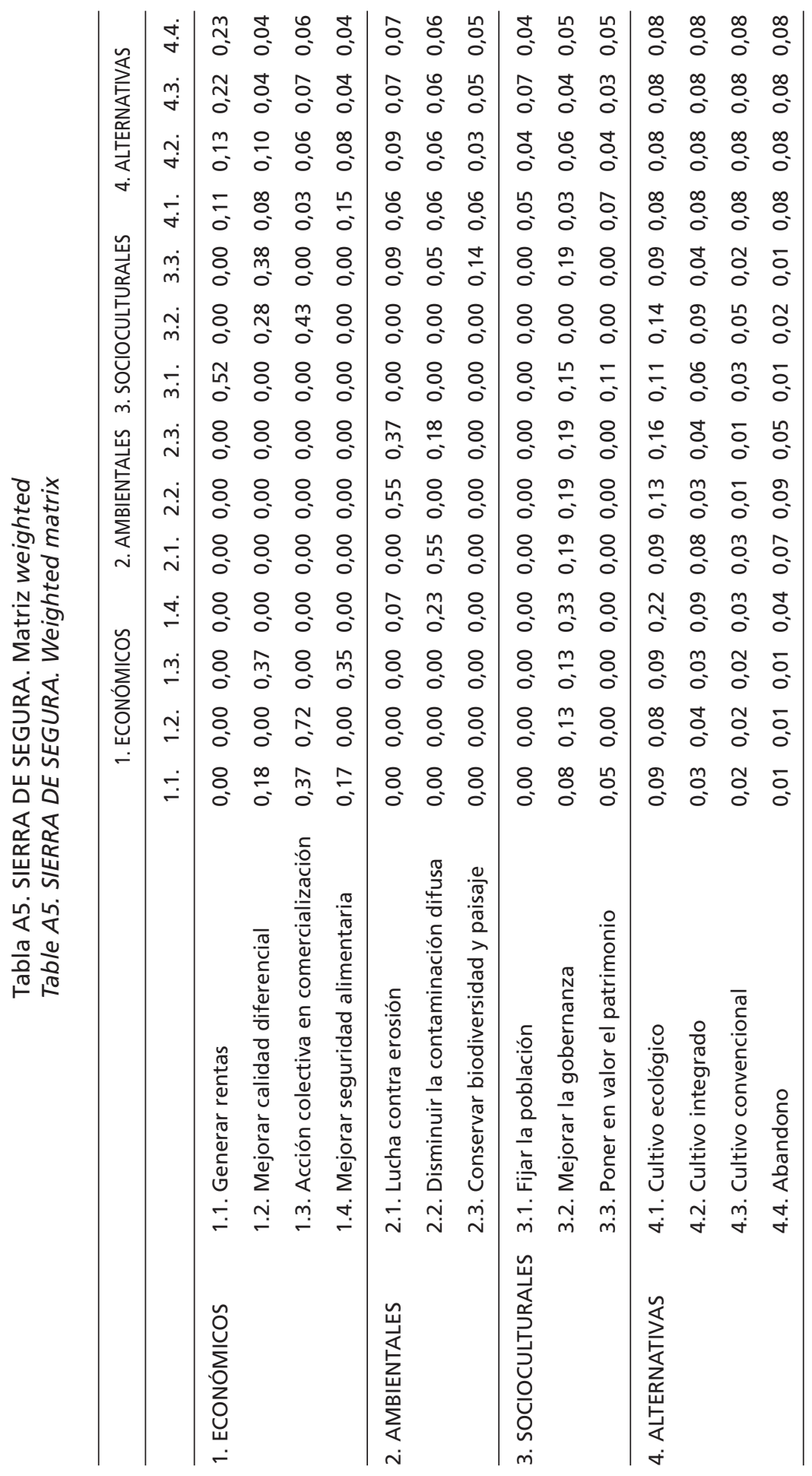




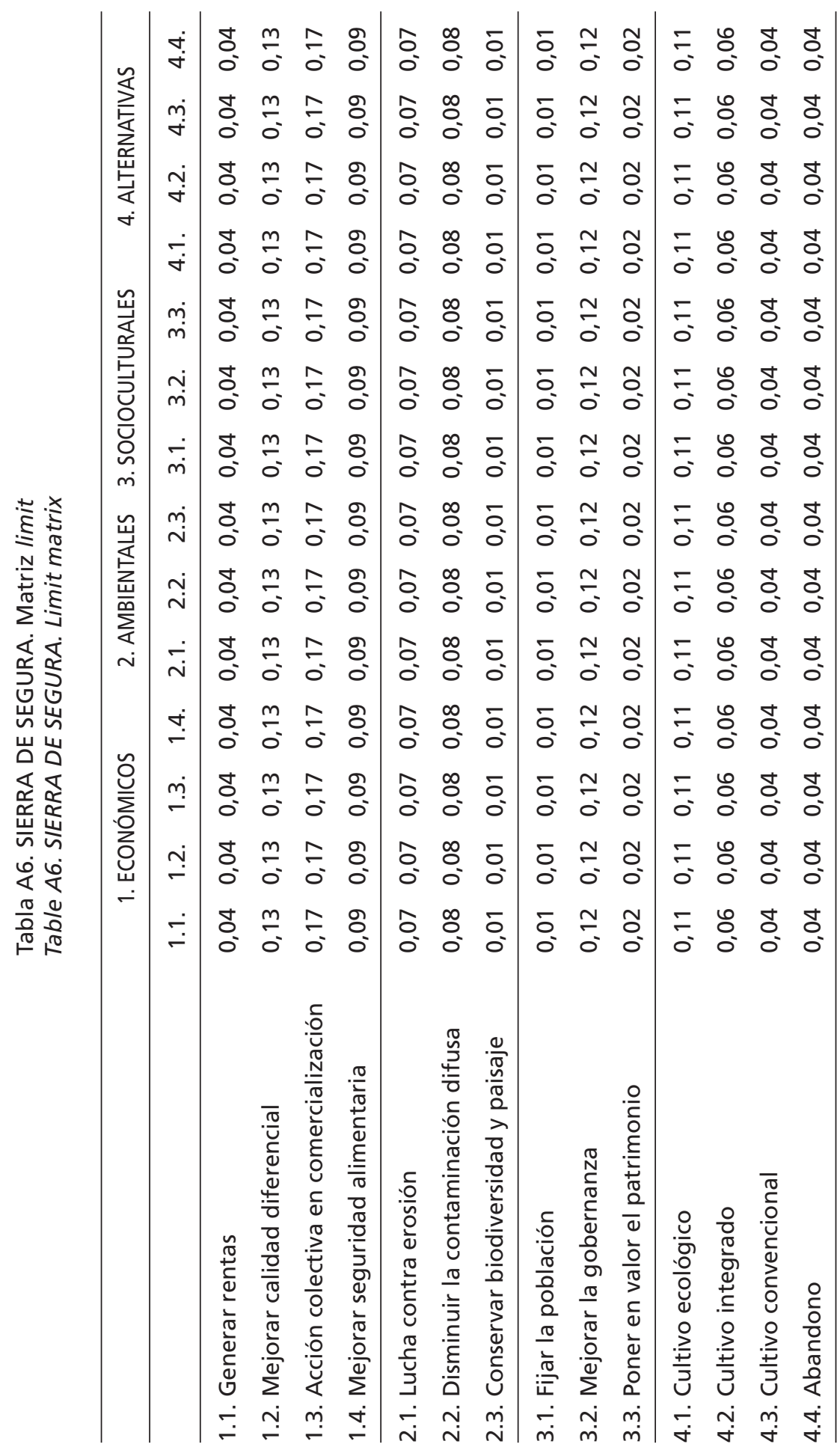




\section{Bibliografía}

Aragonés-Beltrán P, Aznar J, Ferrís-Oñate J, GarcíaMelón M, 2008. "Valuation of urban industrial land: An analytic network process approach". European Journal of Operational Research 185, 1: 322-339.

Arriaza M, Gómez-Limón JA, Kallas Z, Nekhay O, 2008. "Demand for non-commodity outputs from mountain olive groves". Agricultural Economics Review 9, 1: 5-23.

Arriaza M, Nekhay O, 2010. Evaluación social multicriterio del territorio agrícola: el caso del olivar de baja producción. Revista Española de Estudios Agrosociales y Pesqueros 226: 39-69.

Atance I, Tió C, 2000. “La multifuncionalidad de la agricultura: aspectos económicos e implicaciones sobre la política agraria". Estudios Agrosociales y Pesqueros 189: 29-48.

Aznar B, Ferris J, Guijarro F, 2010. "La valoración de inmuebles urbanos mediante el proceso analítico en red". Revista de Estudios Regionales 87: 45-70.

Barjolle D, Réviron S, Sylvander B, 2007. Création et distribution de valeur économique dans les filières de fromages AOP, Économies et Societés, série Systèmes Agroalimentaires 29: 1507-1524.

Cairol D, Coudel E, Knickel K, Caron P, Kröger M, 2009. Multifunctionality of agriculture and rural areas as reflected in policies: the importance and relevance of the territorial view, Journal of Environmental Policy \& Planning 11, 4: 269-368.

Carmona C, Parra C, Sayadi S, Hinojosa A, 2011. "Multifunctional Impacts of the Olive Farming Practices in Andalusia, Spain: An Analytic Network Approach Comunicación al EAAE 2011 Congress August 30- September 2, 2011 Zurich, Suiza.

Chang Cw, Wu Cr, Lin Ct, Lin HI, 2007. "Evaluating digital video recorder systems using analytic hierarchy and analytic network processes". Information Sciences 177: 3383-3396.

Cortes-Aldana FA, García-Melón M, Fernández De Lucio I, Aragonés-Beltrán P, 2010. “Medición del grado de alineación de los fines de una universidad con los resultados obtenidos en su relación con el entorno socioeconómico". VII Congreso de Investigación y Creación Intelectual de la Unimet.

Fleskens L, Duarte F, Eicher I, 2009. A conceptual framework for the assessment of multiple functions of agro-ecosystems: A case study of Trasos-Montes olive groves, Journal of Rural Studies 25: 141-155.

García-Melón M, Ferrís-Oñate J, Aznar-Bellver J, Aragonés-Beltrán P, Poveda-Bautista R, 2008. "Farmland appraisal based on the analytic network process". Journal of Global Optimization 42: 143-155.

Gómez-Limón JA, Barreiro J, 2007. La multifuncionalidad de la agricultura en España. Concepto, aspectos horizontales. Cuantificación y casos prácticos, Ed. Eumedia, S.A, Ministerio de Agricultura, Pesca y Alimentación. 303 pp.

Gómez-Limón JA y Riesgo L, 2012. "Sustainability assessement of olive groves in Andalusia: a methodological proposal". New Medit: Mediterranean Journal of Economics, Agriculture and Environment, 11, 2: 39-49.

Hartell JG, 2004. "Pricing Benefit Externalities of Soil Carbon Sequestration in Multifunctional Agriculture". Journal of Agricultural and Applied Economics 36, 2: 491-505.

Kallas Z, Gómez-Limón JA, Arriaza M, Neckay O, 2006. "Análisis de la demanda de bienes y servicios no comerciales procedentes de la actividad agraria: el caso del olivar de montaña andaluz". Economía Agraria y Recursos Naturales 6: 49-79.

Khan S, Faisal Mn, 2008."An analytic network process model for municipal solid waste disposal options". Waste Management 28, 9: 1500-1508.

Köne AC, Büke T, 2007. "An analytical network process evaluation of alternative fuels for electricitiy generation in Turkey. Energy Policy 35: 5220-5228.

Lee H, Kim CH, Cho H, Park Y, 2009. "An ANP based technology network for identification of core technologies: a case of telecommunication technologies". Expert Syst App/ 36, 1: 894908. 
Mann S, Wüstemann H, 2008. Multifunctionality and a new focus on externalities, The Journal of Socio-Economics 37: 293-307.

Meade LM, Sarkis J, 1999. "Analyzing organizational project alternatives for agile manufacturing processes: an analytical network process" Int J Prod Res 37 2, pp. 241-261.

Muchnik J, Sanz-Cañada J, Torres-Salcido G (eds.), 2008. Systèmes Agroalimentaires Localisés, Cahiers d'Études et des Recherches Francophones IAgricultures 17, 6, Special Issue: 509-592.

Nekhay O, Arriaza M, Boerboom L, 2009. Evaluation of soil erosion risk using Analytic Network Process and GIS: a case study from Spanish mountain olive plantations. J. Environ. Management 90, 10: 3091-3104.

OECD, 2008. Multifunctionality in Agriculture: Evaluating the Degree of Jointness, Policy Implications. Paris. 256 pp.

Parra C, Calatrava J, De Haro T, 2005. “Evaluación comparativa multifuncional de sistemas agrarios mediante AHP: aplicación al olivar ecológico, integrado y convencional de Andalucía". Economía Agraria y Recursos Naturales 5, 9: 27-55.

Parra C, Calatrava J, De Haro T, 2007. "A multi-criteria evaluation of the environmental performances of conventional, organic and integrated olive-growing systems in the south of Spain base on experts' knowledge". Renewable Agriculture and Food Systems 22, 3: 189-203.

Parra C, Calatrava J, De Haro T, 2008. "A systemic comparative assessment of the multifunctional performance of alternative olive systems in Spain within an AHP-extended framework". Ecological Economics 64: 820-834.

Parra C, Groot J, Carmona C, Rossing W, 2008b. "Integrating public demands into model-based design for multifunctional agriculture: An application to intensive Dutch dairy landscapes". Ecological Economics 67, 4 : 538-551.

Perrier-Cornet Ph, Sylvander B, 2000. Firmes, coordinations et territorialité. Une lecture économique de la diversité des filières d'appellation d'origine, Economie Rurale 258: 79-89.
Potter C, Burney J, 2002. "Agricultural multifunctionality in the WTOF legitimate non-trade concern or disguised protectionism?". Journal of Rural Studies 8: 35-47.

Promentilla MAB, Furuichi T, Ishii K, Tanikawa N, 2008. "A fuzzy analytic network process for multi-criteria evaluation of contaminated site remedial countermeasures". Journal of Environmental Management 88: 479-495.

Reig E, 2002. "La multifuncionalidad del mundo rural". Información Comercial Española: Revista de Economía 803: 33-44.

Reig E, Aznar J, Estruch V, 2010. "A comparative study of the sustainability of rice cultivation technologies using the analytic network process". Spanish Journal of Agricultural Research 8, 2: 273-284.

Renting $\mathrm{H}$, Rossing WAH, Groot JCJ, Van der Ploeg JD, Laurent C, Perraud D, Stobbelaar DJ, Van Ittersum MK, 2009. Exploring multifunctional agriculture. A review of conceptual approaches and prospects for an integrative transitional framework, Journal of Environmental Management 90: 112-123.

Rossing WAH, Zander P, Josien E, Groot JCJ, Meyer $B C$, Knierim A, 2007. Integrative modelling approaches for analysis of impact of multifunctional agriculture: a review for France, Germany and The Netherlands, Agriculture, Ecosystems and Environment 120: 41-57.

Saaty TL, 2001. Decision making with dependence and feedback: The Analytic Network Process. RWS Publications, Pittsburgh. 370 pp.

Saaty TL, 2005. "Theory and Applications of the Analytic Network Process: Decision making with benefits, opportunities, costs and risk". RWS Publications. 352 pp.

Sanz-Cañada J, Coq-Huelva D, Sánchez-Escobar F, Hervás-Fernández I, 2012. Environmental and territorial problems of the Spanish olive oil sector and priorities for research and innovation: a Delphi analysis; en F. Arfini, M.C. Mancini \& M. Donati (Eds.) "Local Agri-Food Systems in a Global World: market, social and environmental challenges". Newcastle-upon-Tyme. Cambridge Scholars Publishing, 173-193. 
Sanz-Cañada J, Hervás-Fernández I, Sánchez-Escobar F y Coq-Huelva D, 2011. Investigación e innovación en el sector del aceite de oliva en España. Problemas, oportunidades y prioridades de $I+D+i$. Madrid: Digital CSIC y ALENTA/Plataforma Tecnológica del Olivar, 312 pp.: http:// hdl.handle.net/10261/51799

Sanz-Cañada J, Macías-Vázquez A, 2005. Quality certification, institutions and innovation in local agro-food systems: protected designations of origin of olive oil in Spain, Journal of Rural Studies 21: 475-486.

Sanz-Cañada J, Muchnik J (eds.), 2011. Ancrage et identité territoriale des systèmes agroalimentaires localisés, Économie Rurale, numéro thématique, 322: 1-83.

Stobbelaar DJ, Groot JCJ, Makowski D, Tichit M, 2009. Multifunctional agriculture. From farm diagnosis to farm design and institutional innovation, Journal of Environmental Management 90: 109-111.
Wilson GA, 2009. The spatiality of multifunctional agriculture, Geoforum, 40: 269-280.

Wolfslehner B, Vacik H, 2008. "Evaluating sustainable forest management strategies with the analytic Network Process in a Pressure-stateResponse framework". Journal of Environmental Management 88: 1-10.

Yuksell I, Dagdeviren M, 2007. Using the Analytic Network Process in a SWOT analysis: a case study for a textile firm. Inform Sciences 177, 16: 3364-3382.

Zander P, Knierim A, Groot JCJ, Rossing WAH, 2007) Multifunctionality of agriculture: Tools and methods for impact assessment and valuation, Agriculture, Ecosystems and Environment 120: 1-4.

(Aceptado para publicación el 1 de marzo de 2013) 
L. Pérez y Pérez, P. Egea y J. Sanz-Cañada

\section{VALORACIÓN DE EXTERNALIDADES TERRITORIALES EN DENOMINACIONES DE ORIGEN DE ACEITE DE OLIVA MEDIANTE TÉCNICAS DE PROCESO ANALÍTICO DE RED}

Separata ITEA

INFORMACIÓN TÉCNICA ECONÓMICA AGRARIA, VOL. 109 N. 2 (239-262), 2013

http://dx.doi.org/10.12706/itea.2013.015 